\title{
Weight Encode Reconstruction Network for Computed Tomography in a Semi-Case-Wise and Learning-Based Way
}

\section{Hujie Pan}

Shanghai Jiao Tong University https://orcid.org/0000-0001-5458-2147

Xuesong Li ( $\square$ xuesonl@sjtu.edu.cn )

Shanghai Jiao Tong University

Min Xu

Shanghai Jiao Tong University

\section{Article}

Keywords: Classic algebraic reconstruction technology, ART, computed tomography, Weight Encode Reconstruction Network, WERNet

Posted Date: October 28th, 2020

DOI: https://doi.org/10.21203/rs.3.rs-95793/v1

License: (a) (1) This work is licensed under a Creative Commons Attribution 4.0 International License. Read Full License 


\title{
Weight Encode Reconstruction Network for Computed
}

\section{Tomography in a Semi-Case-Wise and Learning-Based Way}

\author{
Hujie $\mathrm{Pan}^{1}$, Xuesong $\mathrm{Li}^{1 *}$, Min $\mathrm{Xu}^{1 *}$
}

${ }^{1}$ School of Mechanical Engineering, Shanghai Jiao Tong University, Shanghai, China, 200240

*: Corresponding authors

Contact information:

Hujie Pan:phj1991@sjtu.edu.cn,

Xuesong Li: xuesonl@sjitu.edu.cn,

MinXu: 


\begin{abstract}
Classic algebraic reconstruction technology (ART) for computed tomography requires pre-determined weights of the voxels for the projected pixel values to build the equations. However, such weights cannot be accurately obtained due to the high physical complexity and computation resources required. In this study, we propose a semi-case-wise learning-based method named Weight Encode Reconstruction Network (WERNet) to co-learn the target voxel values and intrinsic physics of the case in a selfsupervised manner without labeling the target voxel set. With the help of gradient normalization, the WERNet reconstructed voxel set with a high accuracy and showed a higher capability of denoising compared to the classic ART methods. Moreover, the encoder of the network is transferable from a voxel set with complex structures to unseen cases without the deduction of the accuracy. Our method can be applied in tomography-related applications and similar inversion problems even with unclear intrinsic physics.
\end{abstract}


Computed tomography (CT) with a limited number of views is a type of computational imaging approach that has found applications in understanding complex physics, such as turbulence and combustion in three-dimension ${ }^{1-6}$. The most representative CT algorithms in reconstructing the $3 \mathrm{D}$ scalar field from $2 \mathrm{D}$ images are the algebraic reconstruction technique (ART) and its derivations ${ }^{7-9}$. This category of deterministic algorithms requires pre-calculated weight matrices to correlate the pixel and voxel values numerically. By iterating the ART method view-by-view, the difference between the original and projected pixel values is minimized to get the reconstructed voxel set.

Although widely adopted, a critical issue of the ART-based algorithm is that the weight matrices require considerable sizes of RAM of the computer, which may easily go to tens of GBs and exceed practical hardware limitations ${ }^{4}$. A second considerable issue is that the limited computation resources, together with the inadequate domain knowledge, lead to the mismatch of the assumptions of weight matrix calculation and real-world physics, which eventually results in the inaccuracy of the reconstructed $3 \mathrm{D}$ voxels ${ }^{10-12}$. Moreover, the deterministic nature of the ART-based algorithm makes the reconstruction vulnerable to noise and systematic error. Therefore, limited-view CT problems are still not properly solved and utilized with high fidelity.

Deep learning, as its extraordinary capability of feature abstracting and non-linear fitting ${ }^{13}$, can potentially deal with the issues mentioned above. Some attempts have been made to adopt deep learning algorithms in some reconstruction tasks like the sliced absorption spectroscopy, and chemiluminescence reconstruction ${ }^{14-16}$. However, such end-to-end methods (using the projections as the input and reconstructed object as the outputs) potentially deduct the generalization of the model. It limits the scope of the application of the model and makes it almost not possible to be transferred to unseen cases or those with different numerical setups. Furthermore, a typical deep supervised 
learning method calls for a large amount of comprehensive data with properly assigned ground truth ${ }^{17}$, which cannot be easily fulfilled under many conditions. An attempt us made to increase the generalization of the model with a more built-in projection manner, which helps the model learn a more analytical representation from the projections ${ }^{18}$. However, it is still limited by the end-to-end form that requires well-labeled data and fixed setup.

To tackle the issues mentioned above, we propose a semi-case-wise, learning-based method for limited view computed tomography, named Weight Encode Reconstruction Network (WERNet). Unlike the end-to-end methods that compute the 3D voxels directly by 1-stage inference, we build a brand-new architecture that learns the voxel values during the training process. In the WERNet model, distinctive from typical deep learning models, the sought voxel values are co-trained as parameters in the neural network together with the weight matrix (namely the voxel weight encoder) in an unsupervised manner. Gradient normalization was used to distinguish the two types of parameters and stabilize the training process. We then successfully implement the cotraining of voxel values and voxel weight encoder.

The WERNet algorithm shares some similarity with NeRF algorithm for 3D object surface reconstruction ${ }^{19}$ and beam propagation method (BPM) ${ }^{20}$ together with error backpropagation ${ }^{21}$ for refractive index field reconstruction ${ }^{22}$, but they are intrinsically different. The reconstructed 3D light field in the NeRF model was implicitly incorporated in the neural network, while the 3D flame light field (intensity information) is explicitly and quantitatively set as the parameters in the WERNet algorithm. Moreover, the NeRF and BMP with backpropagation both work with predetermined physical process for imaging which are represented by a rendering module and BMP model respectively. While the WERNet model consists of hybrid parameters, including 
explicit voxel value distribution as network parameters and implicit weight encoders that stand for the physics of light propagation and imaging. It is worth mentioning that the flame intensity parameters in the neural network are physically meaningful in our WERNet model while the co-learned voxel weight encoder represent the intrinsic physics implicitly. These parameters are obtained as the neural network is trained, which sheds light on solving similar physical applications with deep learning algorithms aside from using traditional end-to-end algorithms.

By adopting the novel WERNet method, the voxel set is successfully learned with the cosine similarity between the reconstructed voxel set and the reference greater than 0.999. Moreover, the RAM requirement is dramatically reduced since the tremendously sized weight matrix is no longer needed. With the deterministic nature avoided, WERNet has a much better capability of denoising than that of ART-based methods, especially from the background, which yields a sharper and more accurate reconstructed voxel set. Finally, we freeze the pre-trained weight encoder and transfer it to unseen cases to evaluate the generalization capacity of the WERNet model. It is found that the voxel weight encoder is transferable under different conditions, and it is able to achieve comparable performance in the reconstruction with faster convergence, benefiting from the pre-trained and optimizable weight encoder.

\section{Results}

\section{Numerical Setup and Metrics}

To evaluate the WERNet model, we conducted a series of numerical experiments. The domain of interest (where the flame or flow is set) was discretized into a $30 \times 140 \times 30$ mesh with a resolution of $0.5 \mathrm{~mm}$. The view angles (defined as seen in Fig. 1d) were set with a resolution of $11^{\circ}$ surrounding the object starting from the origin $0^{\circ}$. The 
distance from the camera to the object was set to given values around $5800 \mathrm{~mm}$. The pitch angles of the cameras (as defined in Fig. 1d) were set as either $0^{\circ}, 15^{\circ}$, or $-15^{\circ}$.

We verify our method on two simulated flames with different geometries and complexities, as reflected in Fig. 1a. The former is a jet flame with a simpler and more symmetric geometry, and the latter is a turbulent flame that is more disordered and asymmetric. Projections on the camera were simulated from the ground truth with the pinhole model assumption. The WERNet used the projections to reconstruct the flame just like other algorithms, and the reconstruction is then compared with the ground truth in justifying the capacity of the WERNet method.

a)

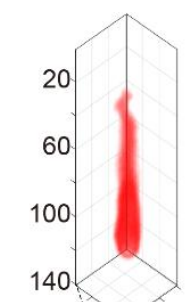

Jet Flame 20 voxels

b)

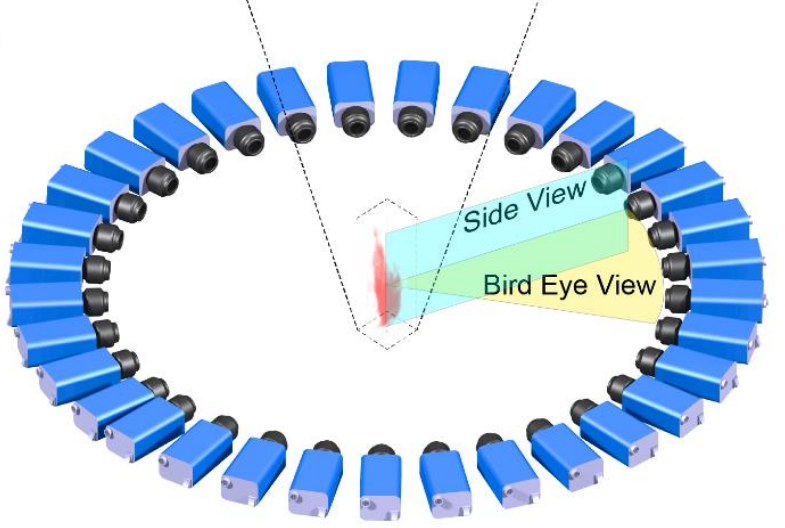

c)
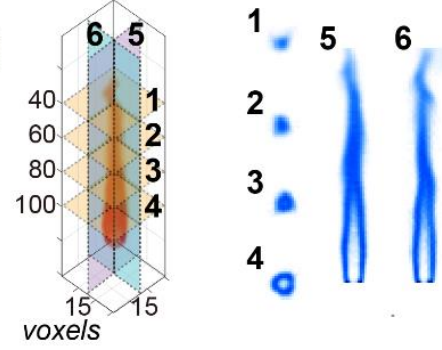

d)

Side view

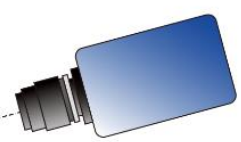

Pitch angle

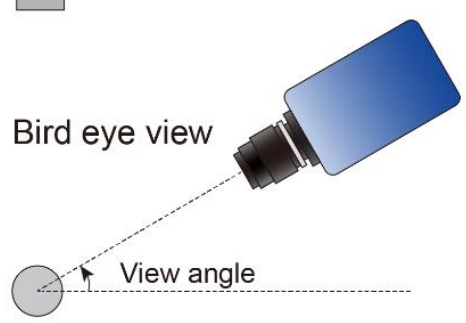

Figure 1. Schematic of the tomographic reconstruction investigated. a) The referent voxel sets of the flames used in the study; b) Layout of the virtual cameras (views); c) Schematics of the crosssectional planes selected; d) Definition of the pitch angle and view angle in this work.

To quantify the discrepancy of the reconstructions, we calculated the cosine similarity between the predicted voxel set and the original one (ground truth) by: 


$$
S_{C}=\frac{\mathbf{v}_{o} \cdot \mathbf{v}_{r}}{\left\|\mathbf{v}_{o}\right\|\left\|\mathbf{v}_{r}\right\|}
$$

in which $v$ is the vector of flattened voxel set. The subscripts $o$ and $r$ represent original and reconstructed voxel sets, respectively. The difference between the reconstructed voxel set and the reference can be represented using cosine distance, which is defined as $D_{C}=1-S_{C}$.

\section{Model Verification and Parametric Study of View Layouts}

We varied the layout of the cameras to capture the multi-view images with different arrangements. For the jet flame voxel set, we conducted three different numerical experiments to test the performance of our model with the same number of views but different poses and distances of the virtual cameras. The first case is with 33 views that uniformly arranged around the object at a constant distance of $5800 \mathrm{~mm}$ from the object. The second case is almost the same as the first one but with a preset pitch angle of $15^{\circ}$ or $-15^{\circ}$. The third case used random object distances on the basis of case 2 for higher complexity.

The cross-sections of the reconstructed flame and their difference with the ground truth are shown in Fig. 2a. For all the three layouts, the WERNet algorithm successfully reconstructed the ground truth of the jet flame. The reconstruction performance is insensitive to pitch angles and the object distances, demonstrating the capacity of the WERNet algorithm. The cosine distances plotted in Fig. $2 \mathrm{~b}$ goes below $10^{-3}$ as the epochs increase to 80 , which quantitatively demonstrates the high accuracy of the reconstructed results.

We further examine the WERNet model with the turbulent flame phantom. The influence of view numbers is explored, as well as their layouts on the reconstruction results. The case with 33 views is designed with a resolution of $11^{\circ}$ for view angles, 15 
and $-15^{\circ}$ alternately for pitch angles, and random object distances ranging from 5500 $\mathrm{mm}$ to $6500 \mathrm{~mm}$. As for the cases with 11 views, we test three different view angle combinations: (1) $0^{\circ}$ to $121^{\circ}$ with a resolution of $11^{\circ}$; (2) $0^{\circ}$ to $220^{\circ}$ with a resolution of $22^{\circ}$; (3) $0^{\circ}$ to $352^{\circ}$ with a resolution of $33^{\circ}$. As shown in Fig. $2 \mathrm{c}$ and $2 \mathrm{~d}$, the WERNet accuracy is sensitive to the view number used but not quite relevant to the view arrangements.

a)

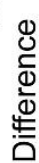
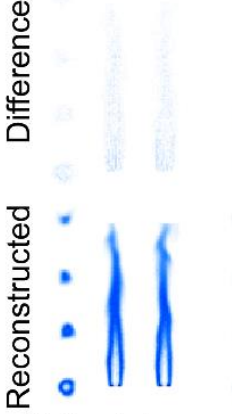

Uniformly Arranged Tilted

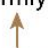

3 Views

c)

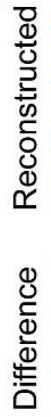

b)

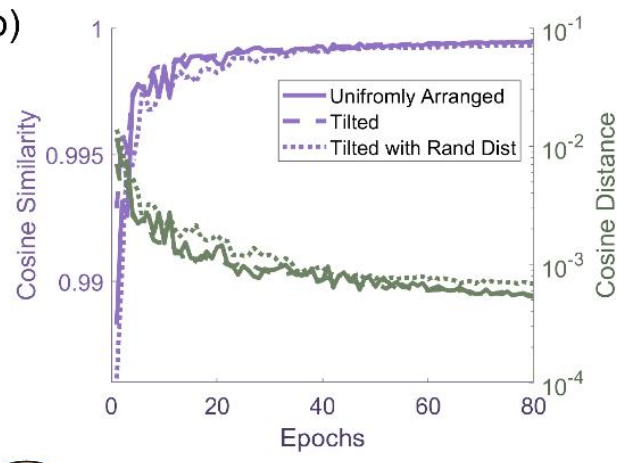

Camera Layout in Bird Eye View

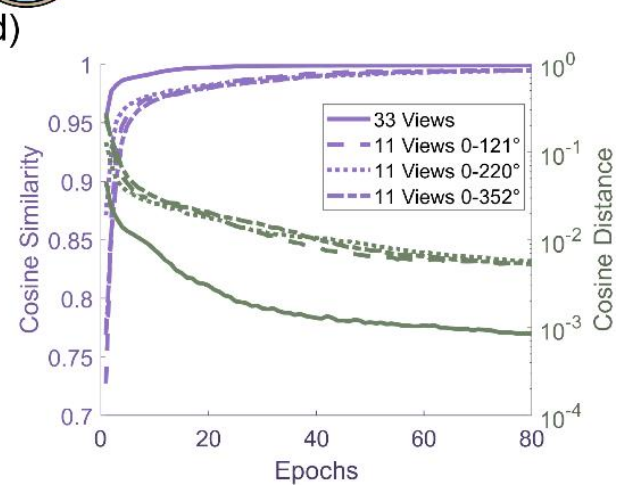

Figure 2. Model performance with different layouts of the views. The rings of different colors

represent the camera layouts in the birds-eye view. a) and c) are the cross-sections of the reconstructed voxel sets of jet flame and turbulent flame, respectively. b) and d) are the cosine similarities of reconstructed result and the reference. The jet flames (upper row) were all reconstructed from 33 views but with different poses and distances of the views (from left to right: constant distance without pitch angle, constant distance with pitch angle, random distance with pitch angle). The turbulent flames were all reconstructed from the views with random distance and pitch angle but has different numbers of views and arrangements. 


\section{Ablation Study \\ Influence of Batch Normalization in the Encoder}

As mentioned in the Method section, the impacting voxel sequences are used as the inputs of the WERNet algorithm. The lengths of impacting voxel sequences of different pixels depend on the actual number of voxels that the pseudo ray passes through. To make the lengths of each voxel sequence consistent, we extended the shorter sequences with a 0 -value tensor. For handling the heterogeneous inputs, we tested three methods to keep the extended part always 0 in the inference so that no gradient will be backpropagated during the training process. Method 1 deactivates the biases of the 1D convolutional layers to make the convolution result of the extended part always 0 . Method 2 activates the bias while using a mask to manually set the encoded voxel weights of the extended part 0 . Method 3 deactivates the bias while adding batch normalization $(\mathrm{BN})$ layers to rescale and shift the features.

As we can see in the results shown in Fig. 3a, the model without biases or BN layers has the worst performance due to the deducted capability of non-linear fittings. Method 2 and 3 achieve a similar level of performance after 40 epochs (with the similarities finally surpass 0.999 ), which indicates an ideal reconstruction result of the voxel set. The equivalent large batch size makes it a good representative of the whole dataset and empowers the $\mathrm{BN}$ layer to stabilize the training process by rescaling the intermediate features and reducing the internal covariate shifts ${ }^{23}$. In that case, the adoption of the BN layer yields a more robust training process than Method 2. 
b) Reconstructed Difference

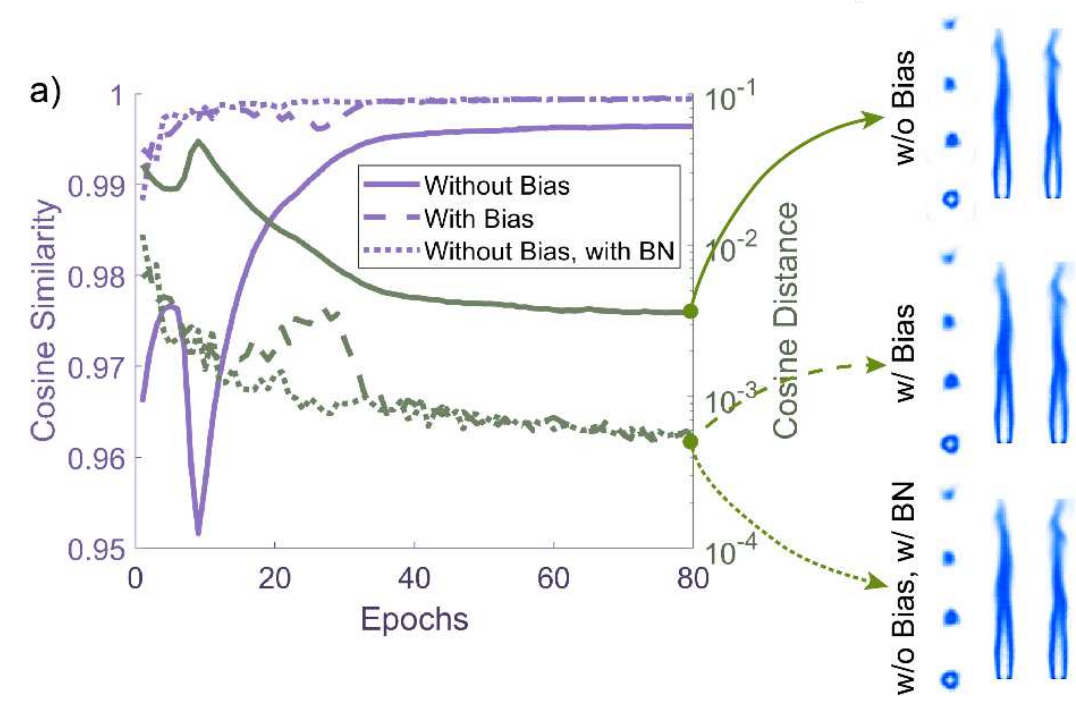

Figure 3. The comparison of different methods adopted in the voxel weight encoder. a) the cosine similarity between the reconstructed results and the reference under different setups. $b$ ) the final reconstructed results and their difference from the reference.

\section{Influence of Gradient Normalization}

In the WERNet network, two categories of network parameters with completely different physical implications were incorporated. The first category is the weight encoder, which represents the physics of light propagation (similar to the weight matrix in traditional CT algorithms but able to summarize more complex non-linear physics). The second category, as we discussed, is the scalar flame intensity distribution itself. Therefore, for this specific application using WERNet, two different gradient flows are needed for co-training.

As mentioned in the Method section, we normalize the gradient for voxel values while keeping the original gradient form for the encoder. The image sequence in Fig. 4a and $4 \mathrm{c}$ show the comparison of the results between the methods with and without gradient normalization. With the original gradient form, the voxel value is trapped after the first ten iterations, which is determined by the first fed batches and the initialization of the voxel weights. Actually, due to the simple multiplication operation of voxel weights 
and voxel value, the updating rate of the voxel value is sensitive to the inferred voxel weights from the encoder. Therefore, the discrepancy of the encoded voxel weight in the early stage can cause the voxel values to deviate from the ground truth significantly. As a result, the original gradient form would yield a slower convergence, and the results might be trapped to local minima.

In contrast, by normalizing the gradient for the training of voxel values, the updating rate of the voxel values is flattened. Therefore, the training process is stabilized. Consequentially, the voxel set with gradient normalization shows an extraordinary convergence speed comparing to that with the original gradient form.

As for the similarity shown in Fig. 4b, gradient normalization significantly outperforms the original gradient form. As seen in Fig. $4 \mathrm{~b}$ and $4 \mathrm{e}$, the method using the original gradient fails to reconstruct the jet flame in 80 epochs. The trend of the similarity curve shows a low likelihood for the method to successfully escape the local minima. On the contrary, the WERNet method that adopts gradient normalization successfully learns the voxel values and voxel weight encoder. 


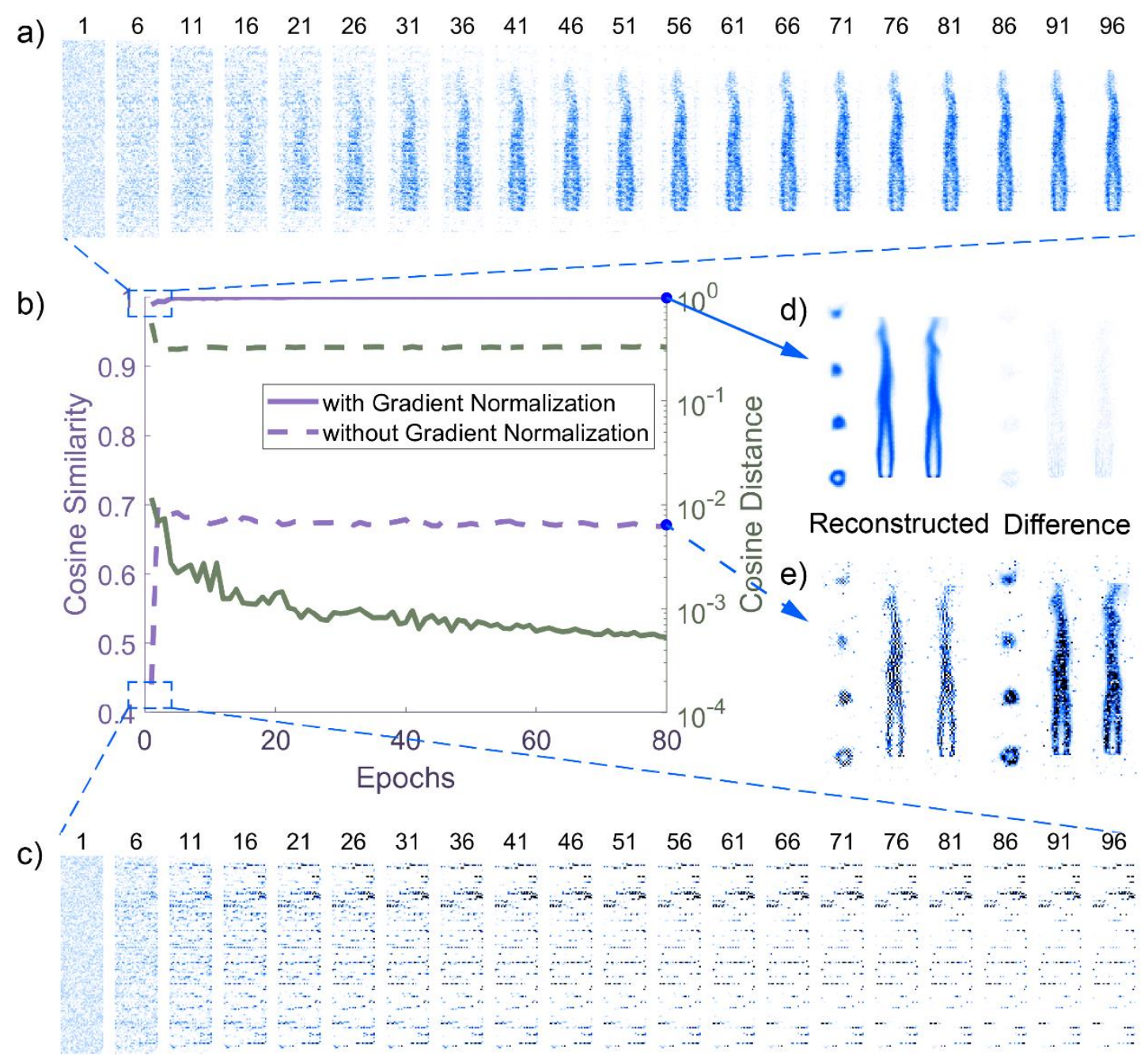

Figure 4. Comparison of original gradient form and normalized gradient backpropagation. a) and

c): First 96 iterations in the first epoch with and without gradient normalization, respectively; b)

Similarity between reconstructed voxel set and the reference; d) and e) are cross-sections of reconstructed voxel sets and their difference from the reference. The upper row shows the result with gradient normalization while the lower row shows the result with original gradient form.

\section{Denoising Capability of WERNet}

To evaluate the capability of the WERNet algorithm in denoising, we add a Gaussian distributed noise to the projection with a magnitude of around $10 \%$ of the maximum of the projected intensities, which is a relatively harsh noise level for reconstruction purposes. Generally, the WERNet method outperforms the classic ART method in reconstructing the $3 \mathrm{D}$ voxel set with noisy projections in both jet and turbulent flame 
cases. For the jet flame, the WERNet method produces the result with minor deterioration in accuracy comparing to the case with clean projections. In comparison, the classic ART method shows a higher sensitivity to the noise added due to its deterministic nature. The flaws of the reconstructed result arise not only in the non-zero region (flame region) but also in the zero-value background of the ground truth.

Compared with the jet flame cases, the voxel value distributions of the turbulent flame are more chaotic, which means the maximum-intensity-based noise is of higher relative magnitudes in turbulent flame cases. The WERNet method still reconstructs the flame with a similarity greater than 0.95 to the ground truth with noise added, while the ART method achieves a performance of around 0.87 only. As seen in Fig. 5b, the WERNet method reconstructs the turbulent flame with higher contrast and less noise in the background comparing to the blurred, noisy result from the ART method.

With more non-zero projected intensities, i.e., valid data for training the voxel weight encoder (otherwise, the gradient would be 0), the reconstructed result converges faster in noisy cases than that without noise, as seen in Fig. 5c. In the case of turbulent flame, due to the noisier projection comparing to that of jet flame, the similarity converges in less than 10 epochs and then changes minorly as the number of epochs increases. 


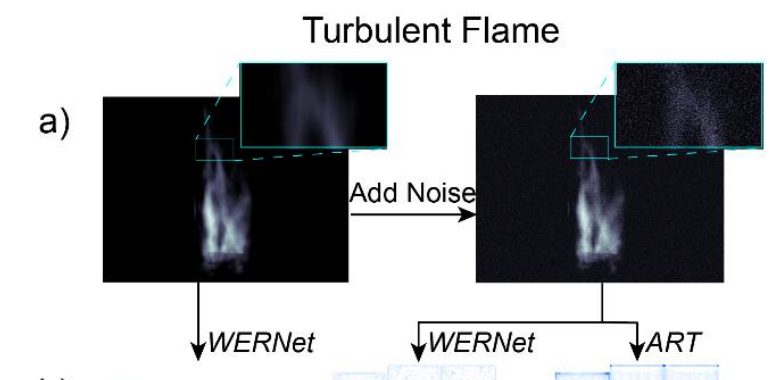

b) 2 .
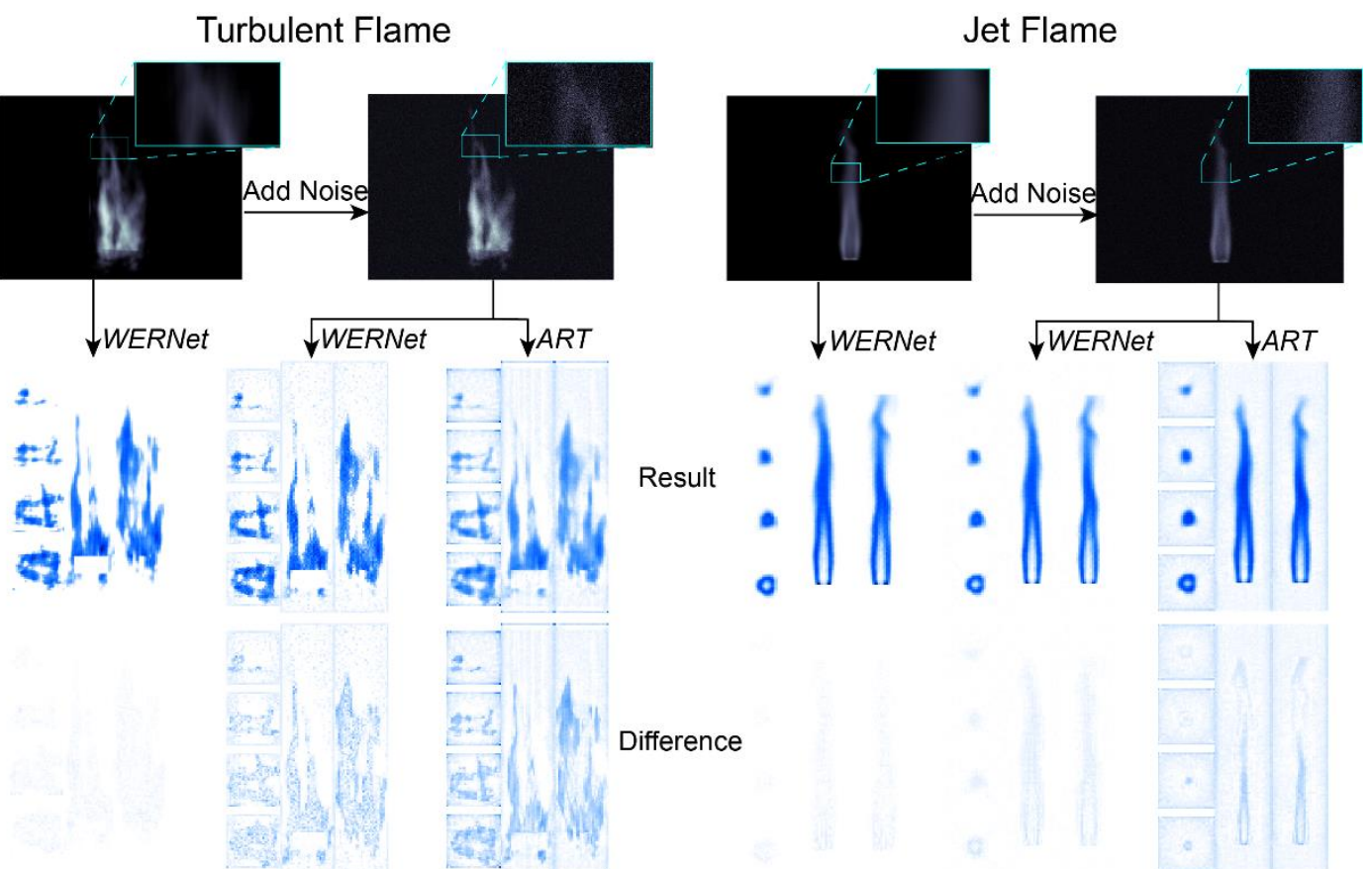

c)
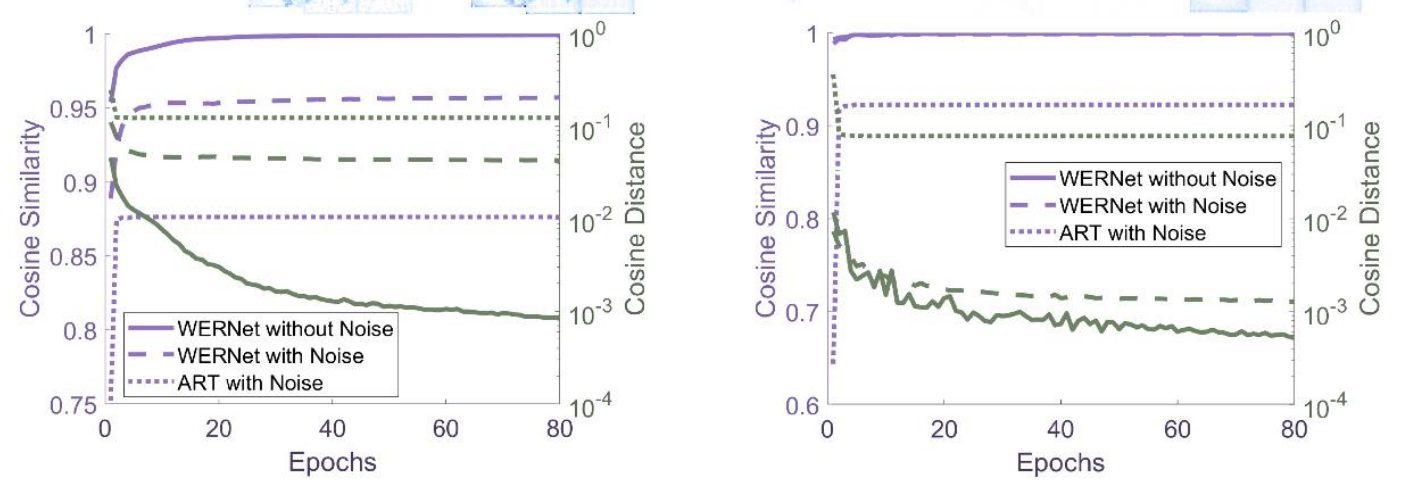

Figure 5. The comparison of the capability of denoising between the ART method and WERNet.

a) clean and noisy projections. b) the cross-sections of reconstructed results using WERNet and

ART methods and their difference from the reference. c) the cosine similarities of the reconstructed results and the reference.

\section{Model Transfer}

As discussed in the above sections, the WERNet method successfully learns the intrinsic nature of light propagations and imaging while accurately reconstructing the voxel set as the model is being trained. The parameters in the network, as a reminder, are flame intensities and weight encoders, respectively. The flame-intensity-related parameters are case-dependent and not transferrable to other cases. While with no change of the physics in producing the projection of the reconstructing region, the 
weighted encoder is intuitively transferable to different cases.

To examine this assumption, we freeze the voxel weight encoders obtained previously and only modify the value of the voxel set, which is randomly initialized. With the voxel weight encoder trained in the jet flame case, the turbulent flame is successfully reconstructed with a final similarity close to 0.99 to the reference after 80 epochs. While when we transferred the weight encoders from the turbulent flame cases to the jet flame, the final similarity stops increasing at 0.92 , as seen in Fig. 6 b. Besides, neither of the results reaches the similarity that achieved in the case with trainable voxel weight encoder $(>0.999)$.

In the investigations we conducted previously, it should be noted that zero value pixels or voxels have a very low contribution to the model training process. Therefore, for the transferred weight encoders to function, non-zero pixel and voxel values should be used during the training. When transferring the encoder of the non-homogeneous biased turbulent flame, the reconstructed jet flame becomes more asymmetric comparing to the reference. However, since the jet flame is relatively rotationally symmetric, which agrees with the isotropic manner of voxel weight derivation in our study, the reconstructed turbulent flame using the jet flame's encoder reaches a higher similarity and the difference of it from the reference evenly distributed in the non-zero voxel region.

To further examine the hypothesis, we built a voxel set with randomized intensities, as seen in Fig. 6a. The random intensity filled the volumetric domain of interest (where the flames are expected to occur) to train the WERNet and then transferred to the reconstruction tasks. The encoder shows an extraordinary capability of generalization. The accuracy of the reconstructed result even outperforms the original WERNet model without weight encoder transfer. Moreover, the voxel set converges in less than 40 
epochs in comparison to 80 epochs in the original WERNet model.

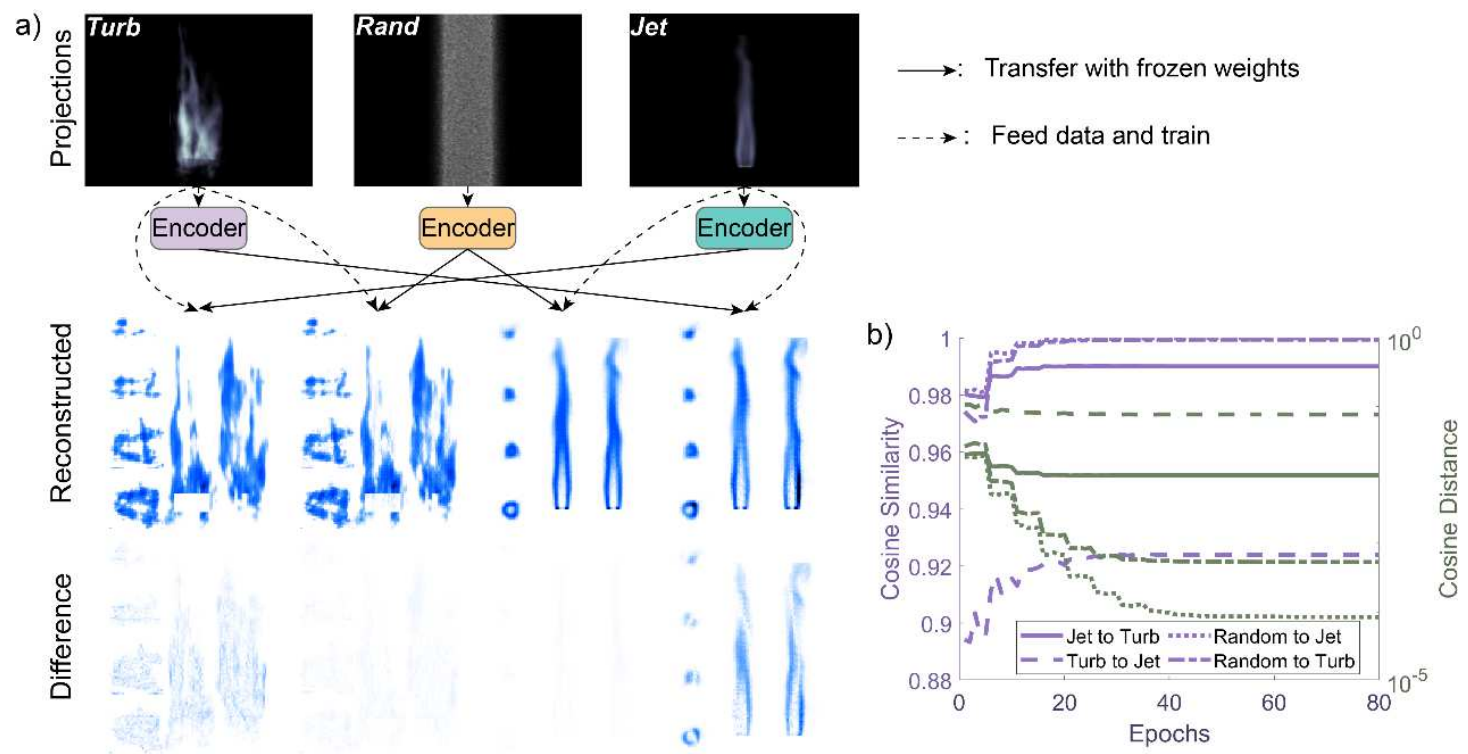

Figure 6. Test result of model transfer of WERNet. The encoders are trained with the projections from turbulent flame, randomized voxel set, and jet flame shown in the first row of a) and then froze and fed with the projections of other cases for transferability test. The second and third rows of a) contain the reconstructed result and their difference from the reference. b) the cosine similarity of the results of different transferring cases and the reference at different epochs.

\section{Discussion}

In this investigation, we proposed a brand-new neural network architecture named WERNet for limited-view computed tomography applications. The WERNet algorithm is a unique algorithm that deposits the sought quantity explicitly in the neural network, and the sought light field/ flame intensity distribution is obtained as the training process is implemented without interpreting the implicit parameters in the network.

Essentially, the WERNet algorithm can function well because the limited view tomography is not an overfitting problem, and there is much more training data (each ray/pixel can be considered as one piece of training data) than the parameters in the neural network. Therefore, the values of the parameters are well constrained by the 
training data. Although the weight encoder works as a black box, the $30 \times 140 \times 30$ flame intensity data is trained with a clear physical meaning. As a result, gradient normalization also works in the WERNet model because of the hybridization of two distinctive types of model parameters. Finally, against the intuition, we are not interested in the inputs (impacting voxel sequence) and the outputs (resultant pixel values) from the model comparing with end-to-end or other supervised models, but finding the sought results back in the trained neural network.

Furthermore, the WERNet model can potentially serve in complex realities that only the ray can be traced, but the voxel weight can hardly be obtained. This occurs in the conditions of non-uniform and translucent medium, which deducts the light intensity along the path, and the attenuation of the translucent signal source is influenced by the voxel intensities along the light path, etc.

Finally, the WERNet model is a semi-case-wise model, as the case-wise part refers to the actual flame intensity distribution and cannot be transferred to other applications, and the non-case-wise part refers to the weight encoders that describe the physics of the light propagation and imaging system. Therefore, this WERNet model is more general and powerful compared with the end-to-end algorithms and backpropagation-asoptimization methods in the existing literature. The hybrid parameter scheme is also valuable in other applications when end-to-end algorithms fail to perform satisfyingly or the exact physics of the case is unclear. This feature of the WERNet model potentially can also be applied for other theoretical or complex equation solving cases, such as predicting Navier-Stokes characteristics in fluid dynamics or stress analysis in solid mechanics both for experiments and numerical applications. We expect the formation of the WERNet algorithms can be applied to a broader range of engineering and fundamental science problems besides the limited-view computer tomography shown 
in this investigation.

\section{Methods}

The WERNet model is a two-step algorithm, including data preparation and model training. The data preparation section introduces how we set the case and preprocess the data to feed WERNet for training.

\section{Data Preparation}

To generate the data with the reference for training and test, we adopt the classic computational photographic method to project the reference voxel set to different angles of views with a pre-determined weight matrix. More specifically, in this work, we used the pinhole model for the ray-calculating purpose. It will not affect the generality of our method since the WERNet does not requires any information of the reference voxel set or its numerical correlation to the projections as the input or initialization. In the current model, each pixel corresponds to one ray only that across the pinhole, while it is readily to establish the correlation between a pixel and multiple rays in the WERNet model.

\section{Impacting Voxel Search}

Unlike a typical end-to-end method that builds the correlation between the voxel value and pixel values directly using a deep neural network, the WERNet model adopts a sequence of impacting voxels of a pixel as the inputs of the algorithm and the pixel values as the outputs. The weight matrix (representing the imaging physics) and the $3 \mathrm{D}$ light field (flame intensity) were considered as the parameters trained in the model.

As seen in Fig. 7, we employ the ray-tracing method to obtain the impacting voxels. The blue line is the reversing ray coming from a pixel through the aperture. When the 
ray passes through the voxel set, a certain sequence of impacting voxels can be extracted as labeled with green faces in Fig. 7. Each impacting voxel and the reversing ray intersect at a segment. We record the coordinates of the center (seen as red points in Fig. 7) of the segment for further feature abstracting. With the above procedure, we gather the information of the impacting voxel sequence, which implies the ray trace and view angle of the camera. It is worth noting that in this arrangement, the ray can be a complex curve, i.e., impacted by refraction, which makes the WERNet model compatible with more challenging reconstruction tasks than traditional methods.

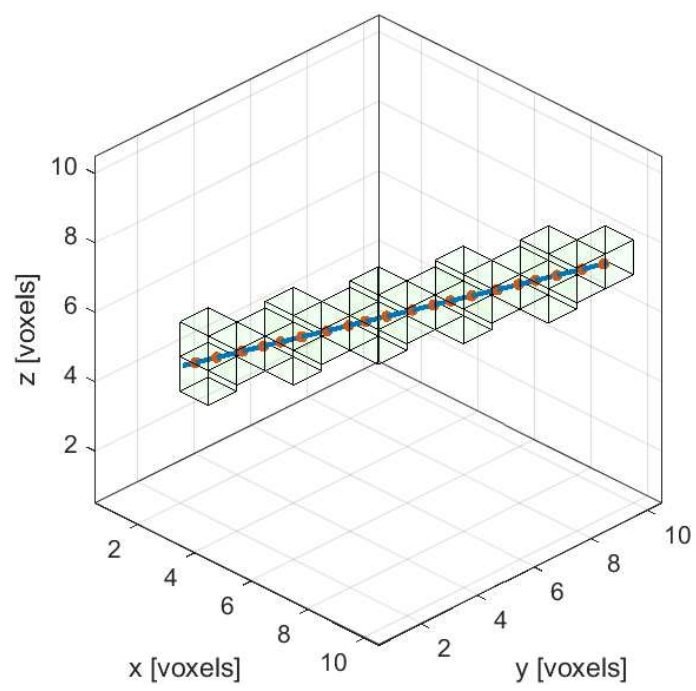

Figure 7, Schematic of voxel search and seg points. The blue line is the pseudo ray and the green cubes are the impacting voxel. The seg points are represented with red points.

\section{Sample Data Generation}

The lengths of the impacting voxel sets that correspond to different pixels can vary. To efficiently feed the data to the WERNet model, we preprocess the data by extending the shorter voxel sequence with a zero tensor and make all the lengths of sequences consistent:

$$
\left[\begin{array}{ll}
\mathbf{I}^{3 \times n} & \mathbf{O}^{3 \times(N-n)} \\
\mathbf{P}^{3 \times n} & \mathbf{O}^{3 \times(N-n)}
\end{array}\right]
$$

in which $\mathbf{I}^{3 \times n}$ represents the indices of the voxels, $\mathbf{P}^{3 \times n}$ is the coordinates of the seg 
points of the voxels, and $\mathbf{O}^{3 \times(N-n)}$ is the zero tensor for the extension. In this manner, the concatenated tensor is of the dimension 3-by- $N$. By doing so, we normalize all inputs to $6 \times N$ for training the WERNet model.

\section{Structure of the WERNet Model}

\section{Architecture}

The architecture of the WERNet model is organized into two sections. The first is the weight encoder section, as seen in the upper region of Fig. 8. For each block the ray passes through, we use a 1-D convolution layer to abstract the features of the inputs and Leaky ReLU for non-linearity. The kernel size of the convolution layer is 3, and the output channel 32. We also set the padding and stride both 1 to make the length of output the same as the input. These parameters have been tested and optimized. After outputting the 1-D tensor of the same dimension with the voxel value, we square the result to make sure that the weights are always non-negative.

The other section is for voxel value learning and pooling, shown in the lower portion of Fig. 8. We determine the impacted voxels using the indices obtained from the target ray information. Then we pool the voxels by extracting these voxels from the voxel set for further computation. Finally, we conduct an element-wise multiplication of voxel weights and voxel values and then sum them up to obtain the predicted pixel value of the projection. 


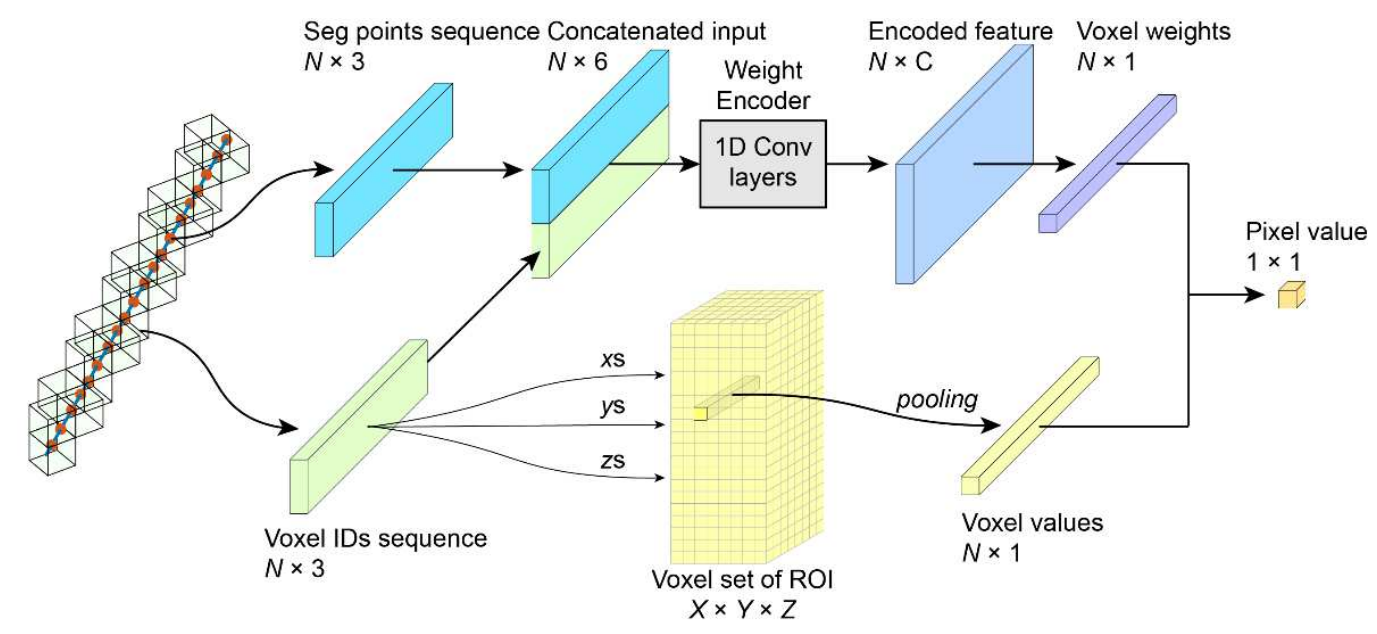

Figure 8: Architecture of the WERNet. The cuboids in the figure represent the tensors of different dimensions and the arrows represent the direction of the data flow. The input of the network is the IDs of the impacting voxel sequence and the coordinates of the seg points of the voxels. The output is the projected pixel value. The network consists of two branches including the voxel weight encoder (upper half) and the voxel value attention (lower half). The voxel weights and voxel values from the two branches are of the same dimension. The final pixel value is obtained by calculating the inner product of the voxel weights and values.

\section{Gradient Normalization}

For the section of calculating the pixel value mentioned previously, we modified the gradients of the voxel value in the following form:

$$
\begin{aligned}
& g_{i, \text { output }}^{v, i}=g_{i, \text { input }}^{v, i} \cdot w_{i} / \operatorname{norm}(\mathbf{w}) \\
& g_{i, \text { output }}^{w, i}=g_{i, \text { input }}^{w, i} \cdot v_{i}
\end{aligned}
$$

in which $g_{i, o u t p u t}$ is the output gradients, $g_{i, \text { input }}$ is the input gradient from the last backpropagation step. $w_{i}$ or $v_{i}$ is the multiplication coefficient of the $i$ th voxel value or weight. For the voxel weight, we maintain the original gradient form to keep the training manner for the weight encoder. As for the voxel value, we add norm(w) to normalize the original gradient to stabilize the training process. 
The modified gradient method proposed offers different optimization schemes for voxel values and the weight encoder, which plays an essential role in remedying the model. More detailed results of the comparison between modified and unmodified gradient form can be found in the results section.

\section{Learning Algorithm}

We compare Adam and SGD optimizers with different learning rates and choose Adam in the model. For the voxel value, since we use the normalized gradients, we test and set the learning rate of 0.01 to accelerate the convergence. As for the other parameters of the model, we set a learning rate of 0.0005 to avoid numerical instability. Moreover, we set a decay rate of 0.5 for the learning rate for every five epochs to finetune the model.

The batch size of the training process is 32 . Notice that each sample contains 100 pairs of input sequences and targets, which indicates an equivalent batch size of 3200 . With the hyperparameters of the learning algorithm set as above, we train our model for 80 epochs to get the final result.

\section{Reference}

1 Cai, W. \& Kaminski, C. F. Tomographic absorption spectroscopy for the study of gas dynamics and reactive flows. Progress in Energy and Combustion Science 59, 1-31, doi:https://doi.org/10.1016/j.pecs.2016.11.002 (2017).

2 Elsinga, G. E., Scarano, F., Wieneke, B. \& van Oudheusden, B. W. Tomographic particle image velocimetry. Experiments in fluids 41, 933-947 (2006).

3 Floyd, J., Geipel, P. \& Kempf, A. M. Computed Tomography of Chemiluminescence (CTC): Instantaneous 3D measurements and Phantom 
studies of a turbulent opposed jet flame. Combustion and Flame 158, 376-391, doi:https://doi.org/10.1016/j.combustflame.2010.09.006 (2011).

$4 \quad \mathrm{Li}, \mathrm{X} . \& \mathrm{Ma}, \mathrm{L}$. Volumetric imaging of turbulent reactive flows at $\mathrm{kHz}$ based on computed tomography. Optics Express 22, 4768-4778, doi:10.1364/OE.22.004768 (2014).

5 Wiseman, S. M., Brear, M. J., Gordon, R. L. \& Marusic, I. Measurements from flame chemiluminescence tomography of forced laminar premixed propane flames. Combustion and Flame 183, 1-14 (2017).

6 Atkinson, C. \& Soria, J. An efficient simultaneous reconstruction technique for tomographic particle image velocimetry. Experiments in Fluids 47, 553 (2009).

7 Andersen, A. H. \& Kak, A. C. Simultaneous algebraic reconstruction technique (SART): a superior implementation of the ART algorithm. Ultrasonic imaging 6, 81-94 (1984).

8 Gordon, R., Bender, R. \& Herman, G. T. Algebraic reconstruction techniques (ART) for three-dimensional electron microscopy and X-ray photography. Journal of theoretical Biology 29, 471-481 (1970).

9 Jiang, M. \& Wang, G. Convergence of the simultaneous algebraic reconstruction technique (SART). IEEE Transactions on Image Processing 12, 957-961 (2003).

10 Trull, A. K. et al. Point spread function based image reconstruction in optical projection tomography. Physics in Medicine \& Biology 62, 7784 (2017).

11 Zhang, B., Zerubia, J. \& Olivo-Marin, J.-C. Gaussian approximations of 
fluorescence microscope point-spread function models. Appl. Opt. 46, 18191829 (2007).

12 Li, X. \& Lin, M. Capabilities and limitations of 3D flame measurements based on computed tomography of chemiluminescence. Combustion \& Flame 162, 642-651 (2015).

13 LeCun, Y., Bengio, Y. \& Hinton, G. Deep learning. nature 521, 436 (2015).

14 Wei, C., Schwarm, K. K., Pineda, D. I. \& Spearrin, R. M. Deep neural network inversion for $3 \mathrm{D}$ laser absorption imaging of methane in reacting flows. Opt. Lett. 45, 2447-2450, doi:10.1364/OL.391834 (2020).

15 Huang, J., Liu, H., Wang, Q. \& Cai, W. Limited-projection volumetric tomography for time-resolved turbulent combustion diagnostics via deep learning. Aerospace Science and Technology, 106123 (2020).

16 Jiang, Y. et al. CSTNet: A Dual-Branch Convolutional Network for Imaging of Reactive Flows using Chemical Species Tomography. arXiv preprint arXiv:2010.03868 (2020).

17 Goodfellow, I., Bengio, Y., Courville, A. \& Bengio, Y. Deep learning. Vol. 1 (MIT press Cambridge, 2016).

18 Würfl, T. et al. Deep learning computed tomography: Learning projectiondomain weights from image domain in limited angle problems. IEEE transactions on medical imaging 37, 1454-1463 (2018).

19 Ben Mildenhall, P. P. S., Matthew Tancik, Jonathan T. Barron, Ravi Ramamoorthi, Ren Ng. NeRF: Representing Scenes as Neural Radiance Fields 
for View Synthesis. arXiv:2003.08934 (2020).

20 Van Roey, J., Van der Donk, J. \& Lagasse, P. Beam-propagation method: analysis and assessment. Josa 71, 803-810 (1981).

21 Rumelhart, D. E., Hinton, G. E. \& Williams, R. J. Learning representations by back-propagating errors. nature 323, 533-536 (1986).

22 Kamilov, U. S. et al. Learning approach to optical tomography. Optica 2, $517-$ $522(2015)$.

23 Ioffe, S. \& Szegedy, C. Batch normalization: Accelerating deep network training by reducing internal covariate shift. arXiv preprint arXiv:1502.03167 (2015).

\section{Author Contribution}

Hujie Pan proposed the original idea, conceived and conducted the numerical experiments via programing and analyzed the results with the help of the other two coauthors.

Xuesong Li supported the study with the knowledge of classic ART algorithm and the methods of data analysis and visualization.

Min Xu contributes the polish of the idea and the final check of the manuscript. All the authors discussed the results. Hujie Pan and Xuesong Li wrote and polished the manuscript. Xuesong Li and Min Xu supervised the project. 


\section{Competing Interest Statement}

The authors have declared that no competing interests exist. 
Figures

a)

b)

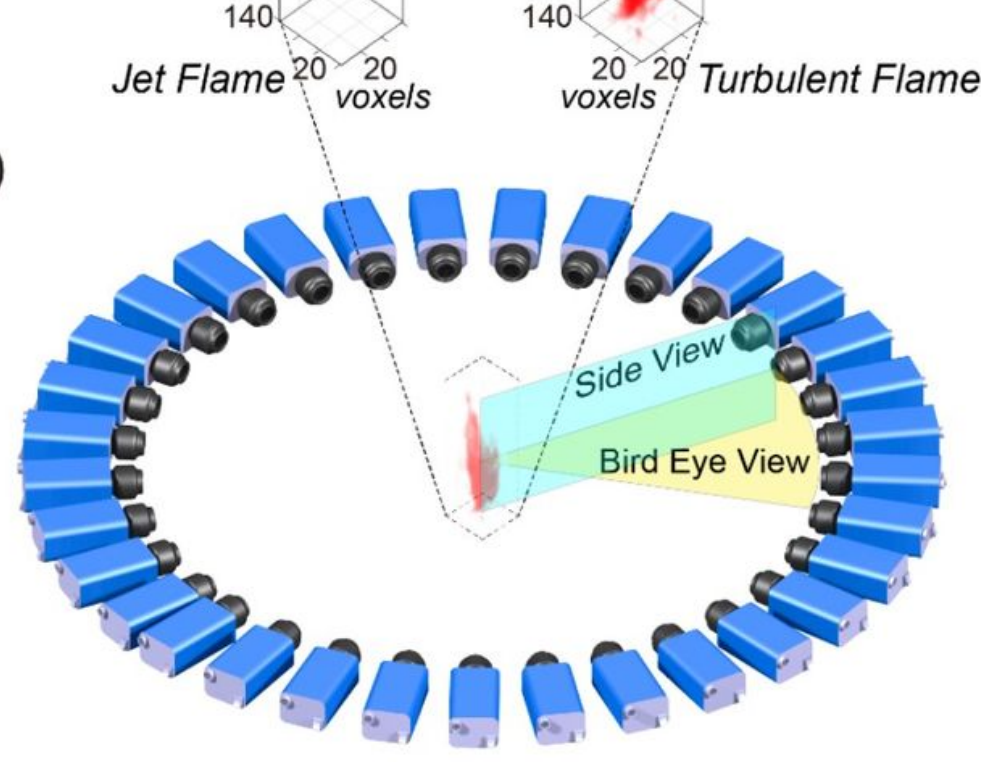

c)
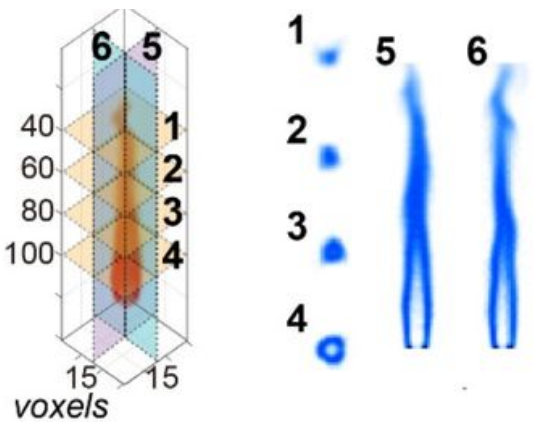

d)

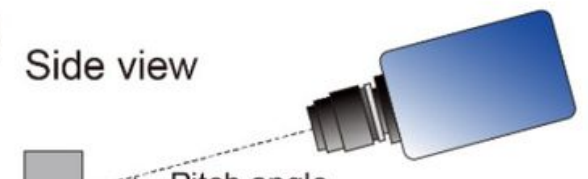

Pitch angle

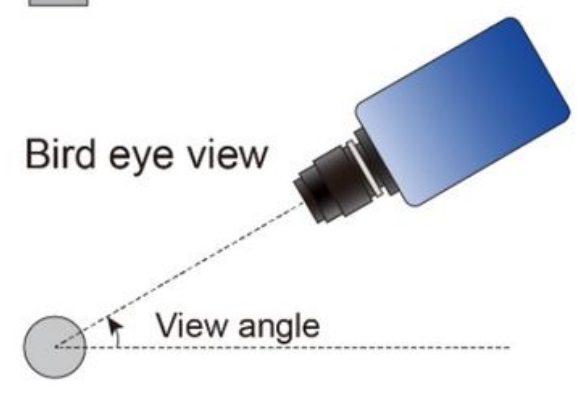

Figure 1

Schematic of the tomographic reconstruction investigated. a) The referent voxel sets of the flames used in the study; b) Layout of the virtual cameras (views); c) Schematics of the cross-sectional planes selected; d) Definition of the pitch angle and view angle in this work. 
a)

$\searrow$
0
$\frac{0}{0}$
$\frac{ \pm}{4}$
$\frac{4}{0}$
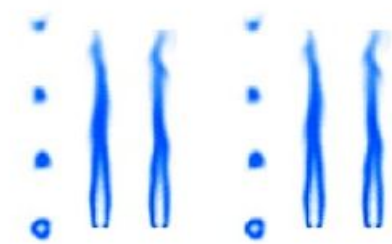

b)

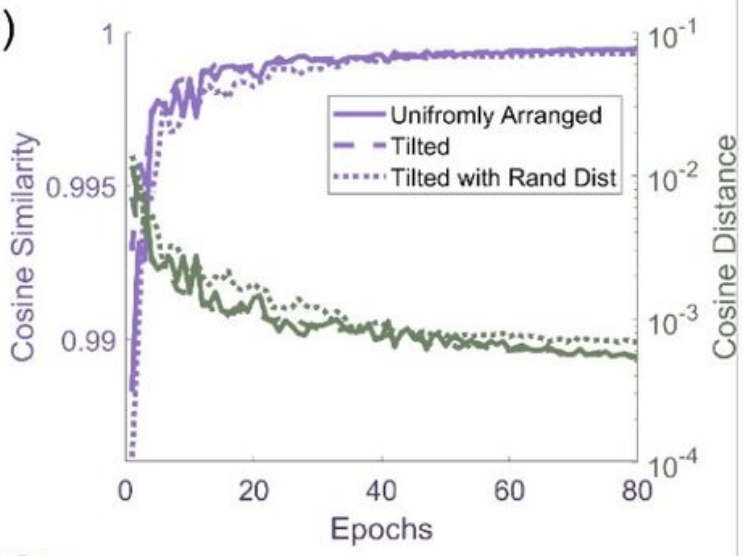

Uniformly Arranged

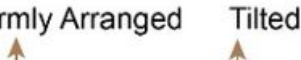

Tilted w/ Rand Dist

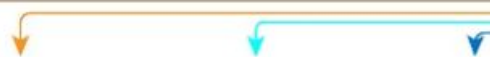

c)

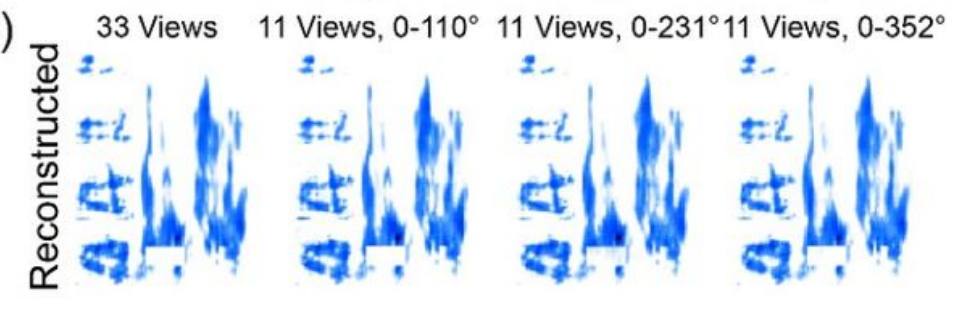

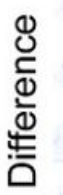

\section{Figure 2}

Model performance with different layouts of the views. The rings of different colors represent the camera layouts in the birds-eye view. a) and c) are the cross-sections of the reconstructed voxel sets of jet flame and turbulent flame, respectively. b) and d) are the cosine similarities of reconstructed result and the reference. The jet flames (upper row) were all reconstructed from 33 views but with different poses and distances of the views (from left to right: constant distance without pitch angle, constant distance with pitch angle, random distance with pitch angle). The turbulent flames were all reconstructed from the views with random distance and pitch angle but has different numbers of views and arrangements. 
b) Reconstructed Difference

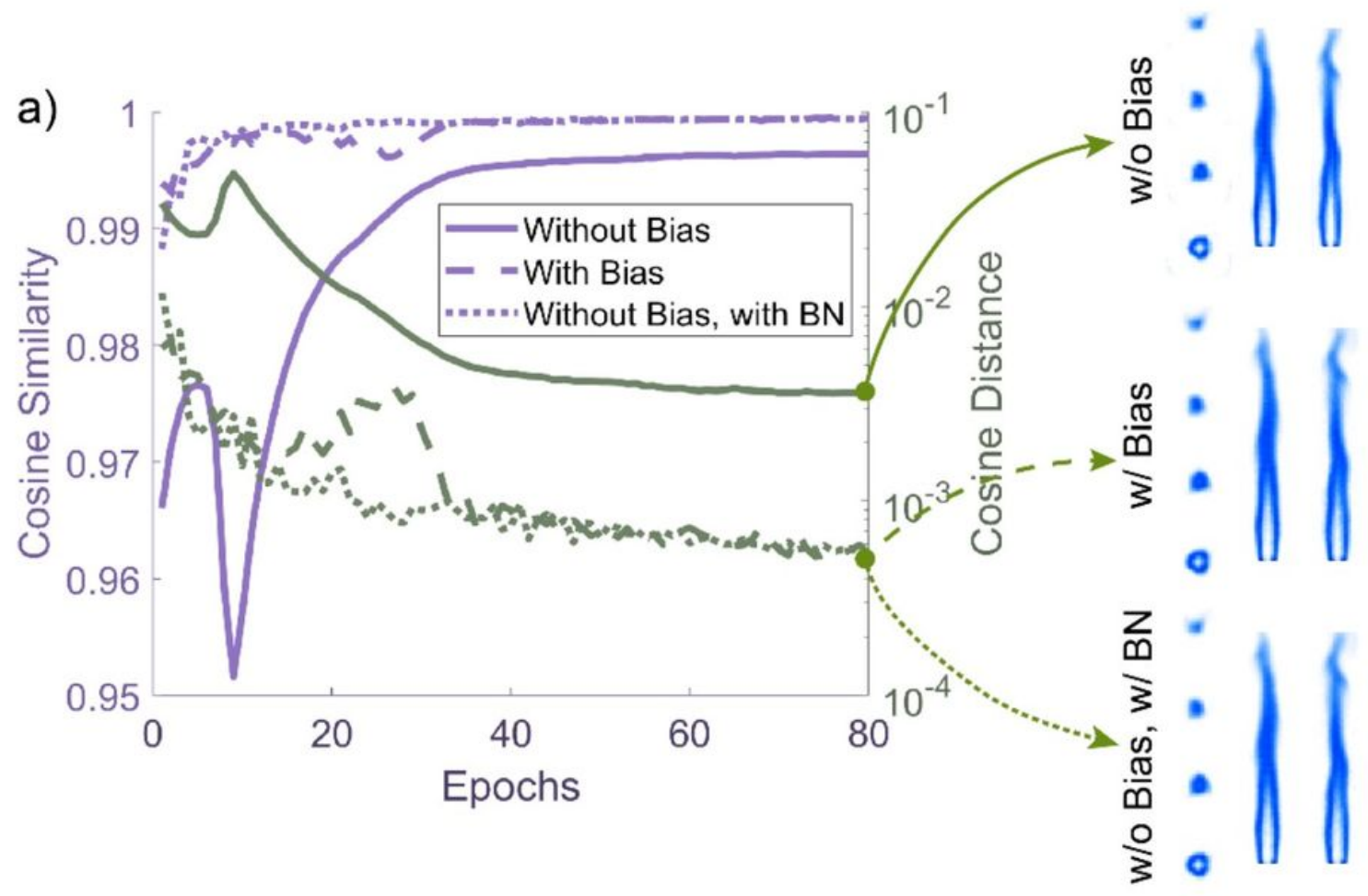

\section{Figure 3}

The comparison of different methods adopted in the voxel weight encoder. a) the cosine similarity between the reconstructed results and the reference under different setups. b) the final reconstructed results and their difference from the reference. 


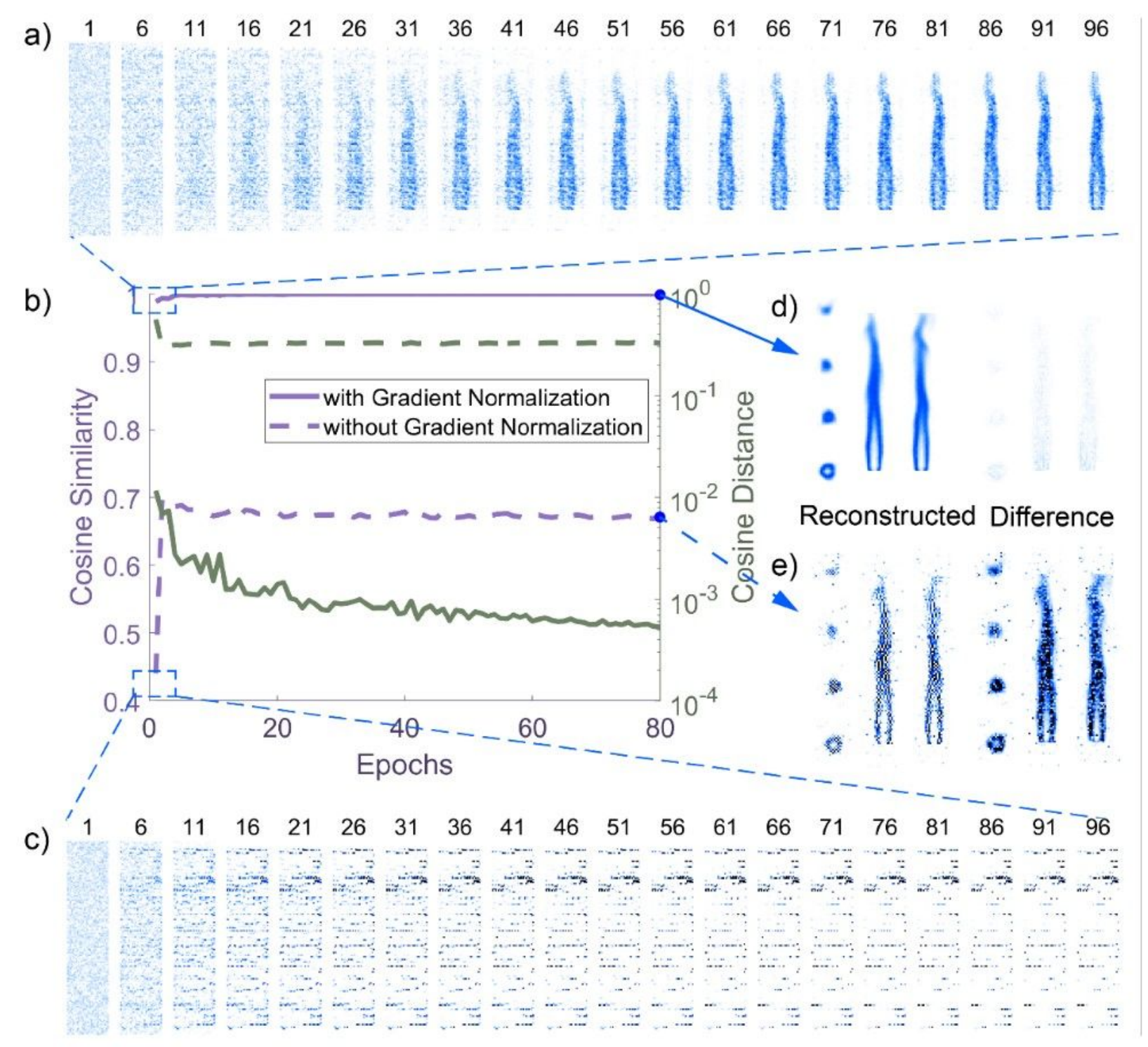

\section{Figure 4}

Comparison of original gradient form and normalized gradient backpropagation. a) and c): First 96 iterations in the first epoch with and without gradient normalization, respectively; b) Similarity between reconstructed voxel set and the reference; d) and e) are cross-sections of reconstructed voxel sets and their difference from the reference. The upper row shows the result with gradient normalization while the lower row shows the result with original gradient form. 


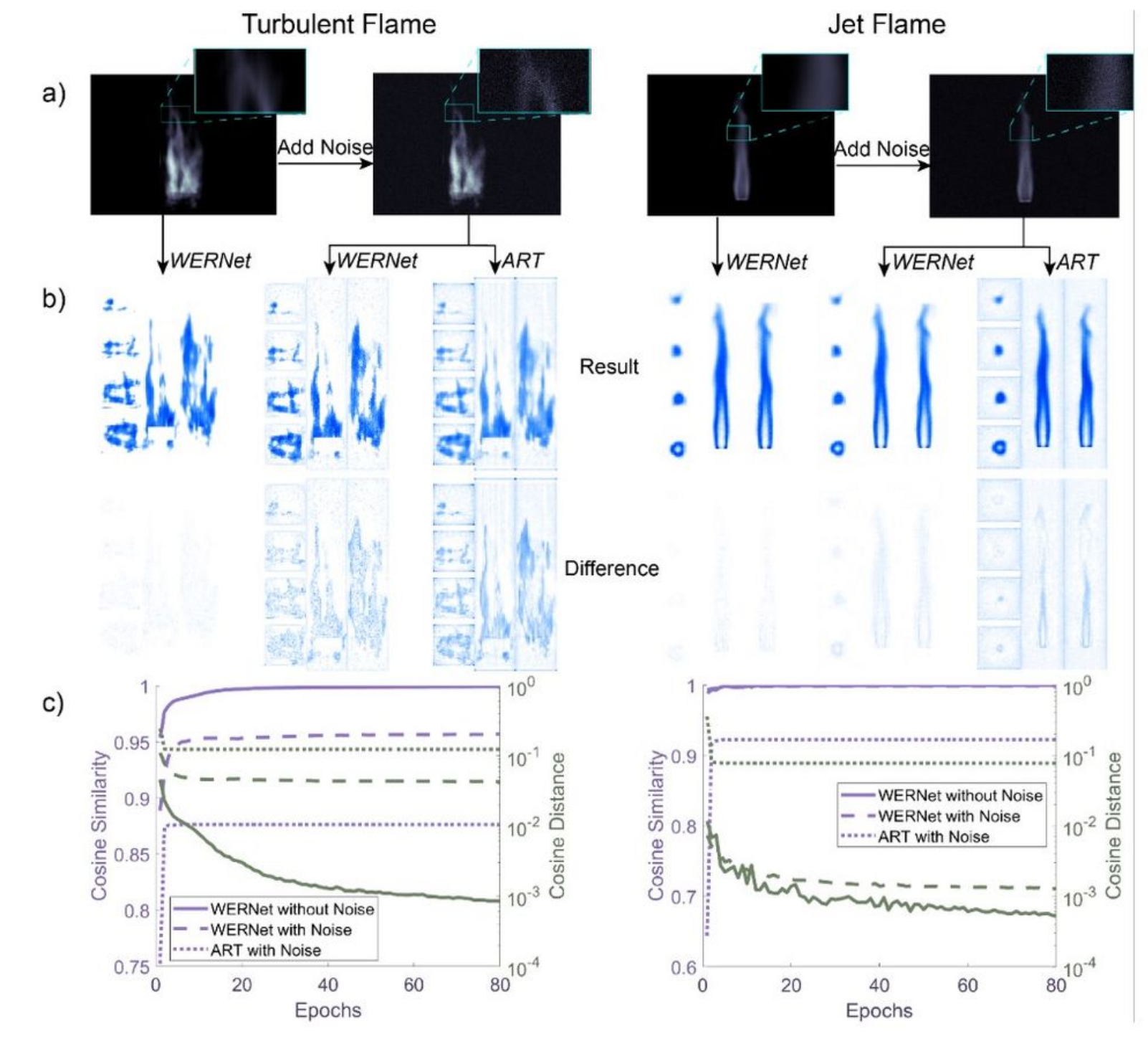

Figure 5

The comparison of the capability of denoising between the ART method and WERNet. a) clean and noisy projections. b) the cross-sections of reconstructed results using WERNet and ART methods and their difference from the reference. $c$ ) the cosine similarities of the reconstructed results and the reference. 

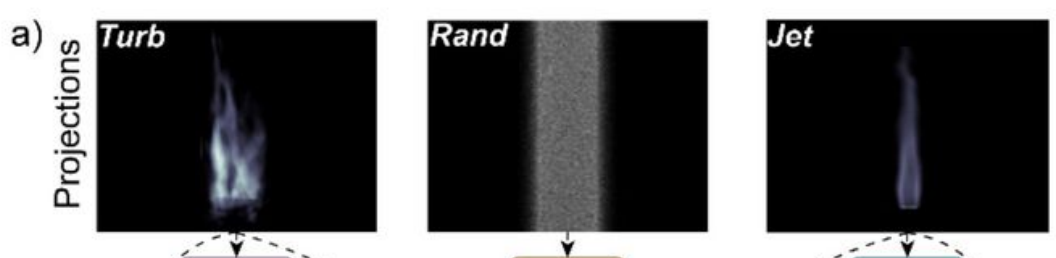

$\longrightarrow$ : Transfer with frozen weights

$\ldots$ : Feed data and train

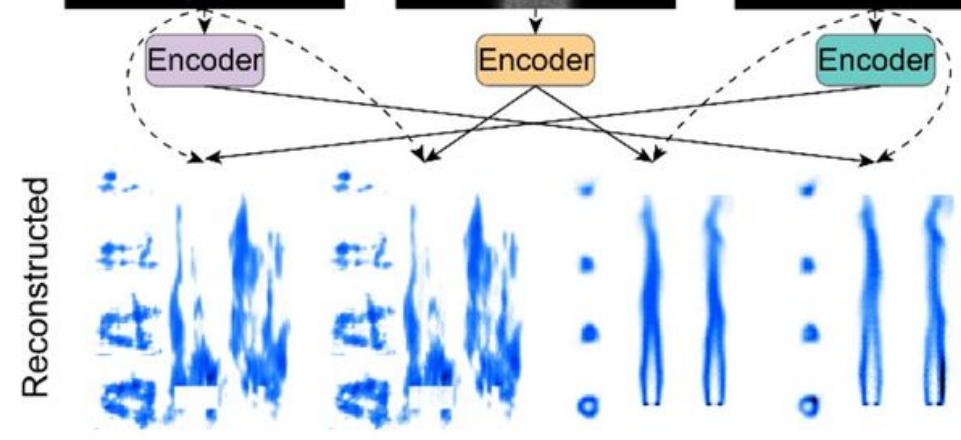

b)

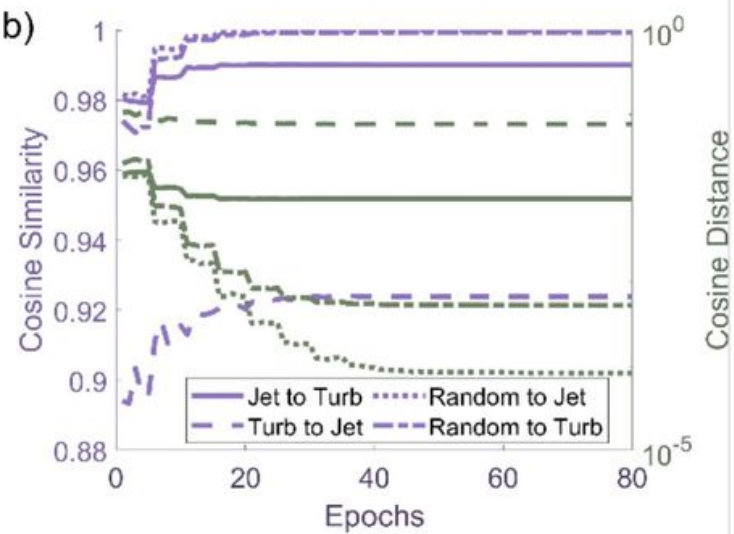

\section{Figure 6}

Test result of model transfer of WERNet. The encoders are trained with the projections from turbulent flame, randomized voxel set, and jet flame shown in the first row of a) and then froze and fed with the projections of other cases for transferability test. The second and third rows of a) contain the reconstructed result and their difference from the reference. $b$ ) the cosine similarity of the results of different transferring cases and the reference at different epochs. 


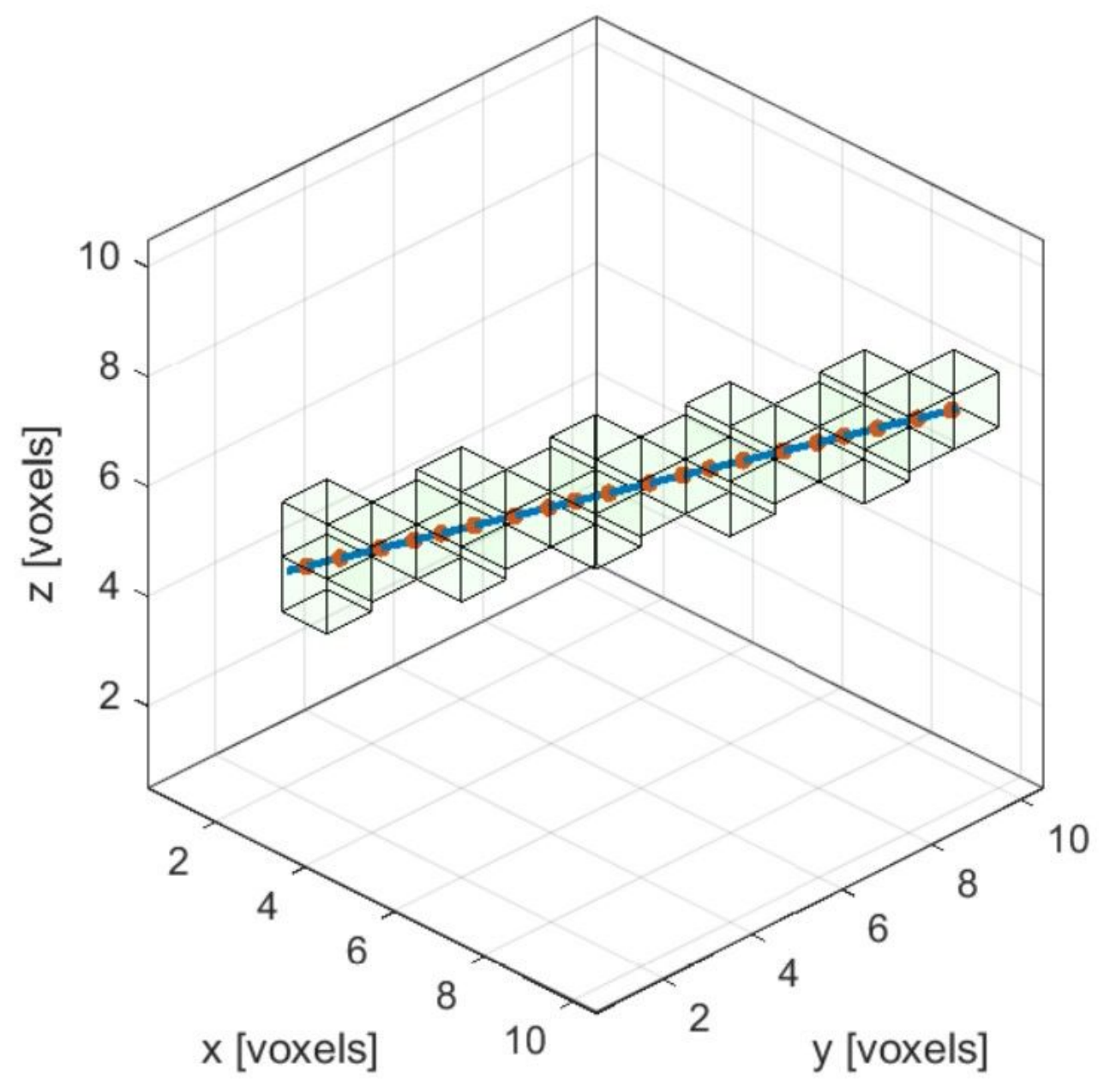

Figure 7

Schematic of voxel search and seg points. The blue line is the pseudo ray and the green cubes are the impacting voxel. The seg points are represented with red points. 


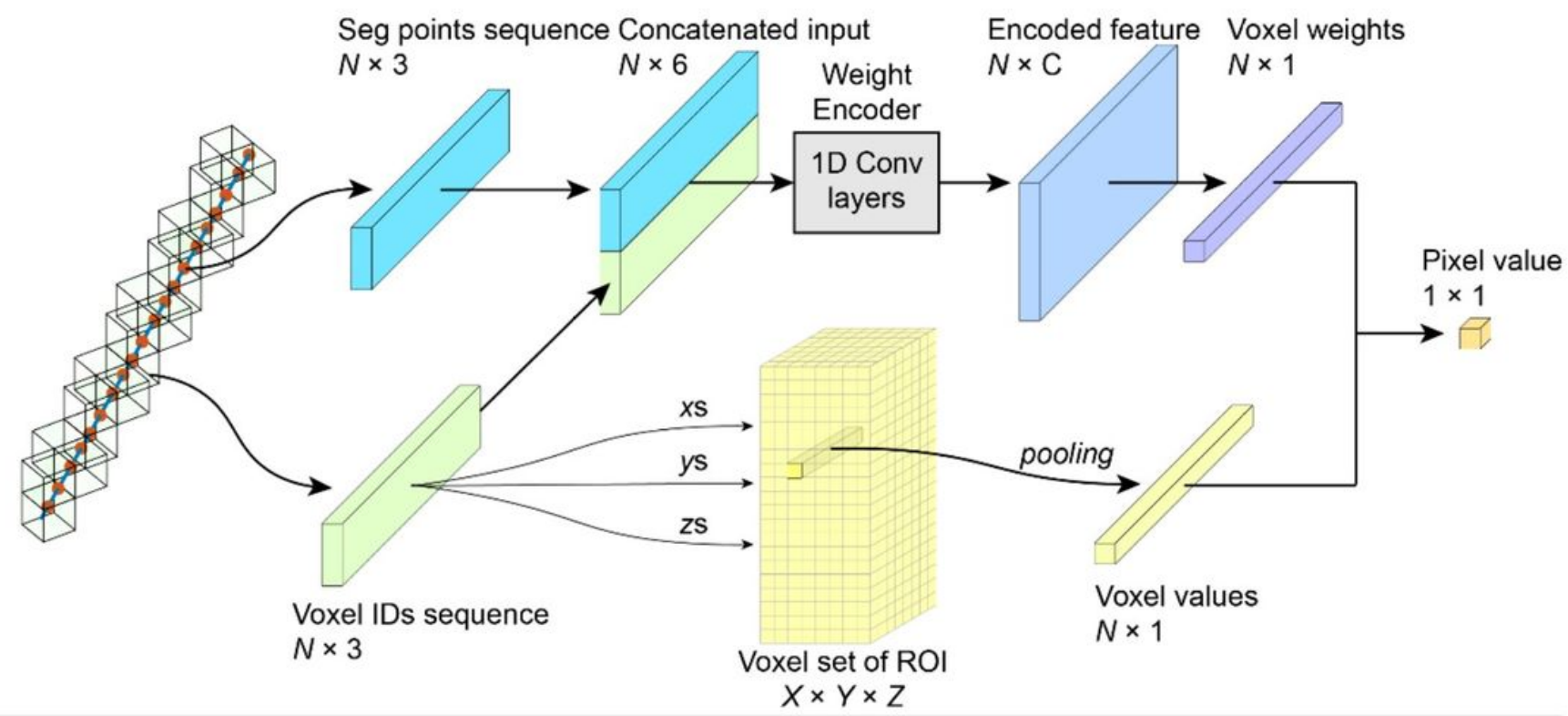

Figure 8

Architecture of the WERNet. The cuboids in the figure represent the tensors of different dimensions and the arrows represent the direction of the data flow. The input of the network is the IDs of the impacting voxel sequence and the coordinates of the seg points of the voxels. The output is the projected pixel value. The network consists of two branches including the voxel weight encoder (upper half) and the voxel value attention (lower half). The voxel weights and voxel values from the two branches are of the same dimension. The final pixel value is obtained by calculating the inner product of the voxel weights and values. 